\title{
Hemorragia de disco óptico possivelmente secundária a descolamento espontâneo do vítreo posterior
}

\author{
Optic disc haemorrhage possibly secondary \\ to posterior vitreous detachment
}

Rodrigo Angelucci', Renata Canovas ${ }^{2}$, Jorge Mitre ${ }^{3}$, José Ricardo Rehder ${ }^{4}$

\section{ResUMO}

Descreve o diagnóstico e conduta em um caso de hemorragia de disco óptico possivelmente secundária a descolamento espontâneo do vítreo posterior. Relata um caso de hemorragia de disco óptico possivelmente secundária a descolamento espontâneo do vítreo posterior, discutindo os aspectos diagnósticos, prognósticos e conduta oftalmológica.Observou-se regressão da hemorragia do disco após $6 \mathrm{~h}$ de evolução do quadro sem sinais de recidiva durante os registros subseqüentes. A Oftalmoscopia com Laser de Varredura mostrou-se como um avançado método alternativo e eficaz no diagnóstico e seguimento do descolamento vítreo.

Descritores: Descolamento do vítreo; Hemorragia retiniana; Disco óptico/irrigação sangúínea; Oftalmoscopia/métodos; Relatos de casos [Tipo de publicação]

\footnotetext{
${ }^{1,2}$ Médicos colaboradores do Setor de Retina e Vítreo da Disciplina de Oftalmologia da Faculdade de Medicina do ABC - FMABC - Santo André (SP), Brasil;

${ }^{3}$ Médico chefe do Setor de Retina e Vítreo da Disciplina de Oftalmologia da Faculdade de Medicina do ABC - FMABC - Santo André (SP), Brasil;

${ }^{4}$ Professor titular, Chefe da Disciplina de Oftalmologia da Faculdade de Medicina do ABC - FMABC - Santo André (SP), Brasil; Doutor, Professor adjunto da Universidade Federal de São Paulo - UNIFESP - São Paulo (SP), Brasil.

Trabalho realizado no Instituto de Olhos da Disciplina de Oftalmologia da Faculdade de Medicina do ABC - SP
} 


\section{INTRODUÇÃO}

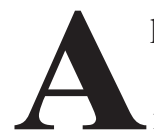
lterações na interface vítreo-retiniana podem acarretar diferentes formas de comprometimento da função visual ${ }^{(1)}$.

O corpo vítreo, constituído basicamente por ácido hialurônico e microfibrilas de colágeno e água, encontra-se firmemente aderido na extrema periferia da retina, formando a base vítrea. No pólo posterior, a hialóide fixa-se na membrana limitante interna retiniana (MLI), formando áreas de maior aderência na região do disco óptico, mácula e arcadas vasculares ${ }^{(1-2)}$.

O descolamento do vítreo posterior (DVP) consiste da associação entre a liquefação vítrea e a separação do córtex posterior vítreo da MLI da retina. Este processo ocorre fisiologicamente com o avanço da idade e mais precocemente nos olhos míopes e facectomizados.

Clinicamente, o DVP se manifesta com sintomas de "moscas volantes" (floaters) e "flashes de luz" (fotopsias) que diminuem com a evolução do quadro. Entretanto, complicações como rupturas e descolamentos retinianos, membranas epirretinianas e hemorragias de disco óptico podem estar associadas ao DVP.

A oftalmoscopia com laser de varredura (OCT/ SLO) é um novo método útil e não invasivo na detecção dos descolamentos vítreos, já que permite avaliar a relação vítreo-retiniana com seus pontos de maior aderência e correlacioná-los com os achados clínicos ${ }^{(3.5)}$.

O objetivo deste trabalho é descrever o caso de um paciente com hemorragia de disco óptico, possivelmente secundária a descolamento espontâneo do vítreo posterior, analisando seus aspectos clínicos e exames diagnósticos.

\section{DesCrição do CASO}

Paciente do sexo masculino, branco, 55 anos, com sintomas de moscas volantes e fotopsias no olho direito há 4 horas. Não há antecedentes pessoais e familiares.

Ao exame apresentava acuidade visual $\mathrm{em}$ ambos os olhos (AO) de 20/20. A pressão intra-ocular era de 15 $\mathrm{mmHg}(\mathrm{AO})$. $\mathrm{O}$ exame biomicroscópico de $\mathrm{AO}$ não apresentava qualquer alteração do segmento anterior. No mapeamento de retina do olho direito (OD) observou-se disco óptico com bordas e coloração normais, escavação fisiológica apresentando hemorragia inferior (figura 1), separação do vítreo posterior observando-se Anel de Weiss (figura 2), sem alterações periféricas. $\mathrm{O}$ olho esquerdo (OE) revelou-se dentro da normalidade.
Foram realizados os exames complementares para elucidação diagnóstica.)

Perimetria visual computadorizada $(\mathrm{CV})$ branco no branco - dentro da normalidade em AO.

Já a oftalmoscopia com laser de varredura (OCT/ SLO) revelou no OD, DVP e no OE corpo vítreo sem alterações (figuras 3 e 4). A tomografia de coerência óptica de disco óptico e mácula revelou-se dentro da normalidade em AO (figuras 5 e 6).

Evolução: Diante do quadro foi feito o diagnóstico de hemorragia de disco óptico, possivelmente secundária a descolamento espontâneo do vítreo posterior, tendo como conduta expectante e acompanhamento do caso com 6 horas, 1 dia, 1 semana e 1 mês de evolução.

Pôde-se observar regressão da hemorragia do disco após 6 horas de evolução do quadro sem sinais de recidiva durante os registros subseqüentes (figura 7).

\section{DisCUSSÃO}

A hemorragia de disco óptico caracteriza-se por uma descompensação e ruptura dos capilares pré-discais. É encontrada com maior freqüência em pacientes portadores de glaucoma primário de ângulo aberto como sinal clínico de descontrole da doença e também nos casos de glaucoma de pressão normal. Com menor incidência, a hemorragia de disco pode estar associada a outras doenças oculares, tais como, neuropatias, trauma contuso e descolamento vítreo posterior, como observado no caso relatado ${ }^{(6)}$.

O DVP raramente requer alguma forma de tratamento que seja diferente do acompanhamento clínico e orientações ao paciente quanto aos riscos de complicações? ${ }^{2}$.

A identificação do DVP pode ser realizada basicamente pela análise do vítreo sob oftalmoscopia indireta ou pela biomicroscopia de segmento posterior com lentes apropriadas de 60 ou 90 dioptrias, além da ecografia modo B, sendo na maioria dos casos suficiente para concluir o diagnóstico. Entretanto, McDonald et al. relataram que existem algumas situações nas quais sintomas de DVP são observados e não são detectáveis ao exame clínico ${ }^{(7)}$. Nesses casos, a OCT/SLO torna-se útil na detecção de pequenos pontos de liquefação vítrea e áreas de aderências vítreo-retinianas anômalas que podem acarretar em complicações como a hemorragia de disco óptico ${ }^{(3)}$.

No caso exposto, o paciente foi assistido logo após o início da sintomatologia, sendo observado o DVP com a presença do Anel de Weiss à oftalmoscopia indireta, 


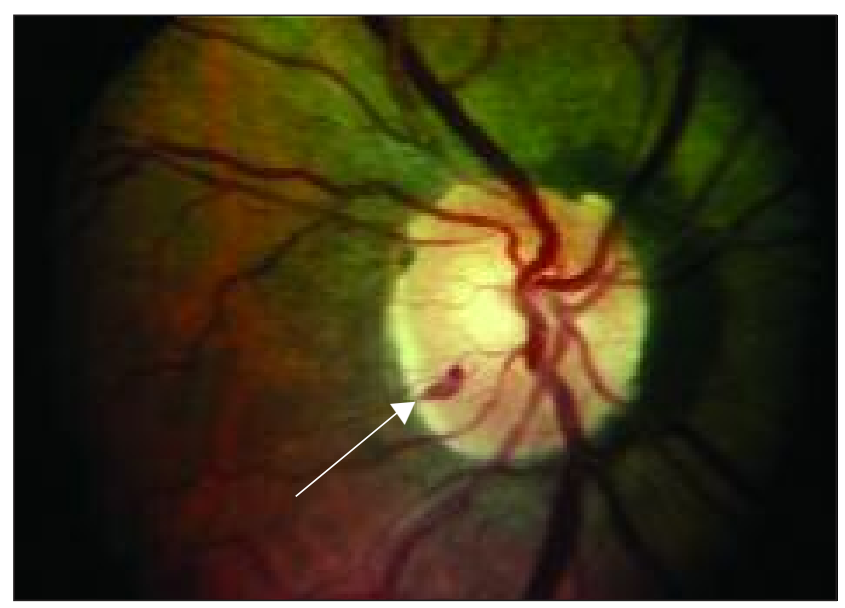

Figura 1: Hem orragia peripapilar

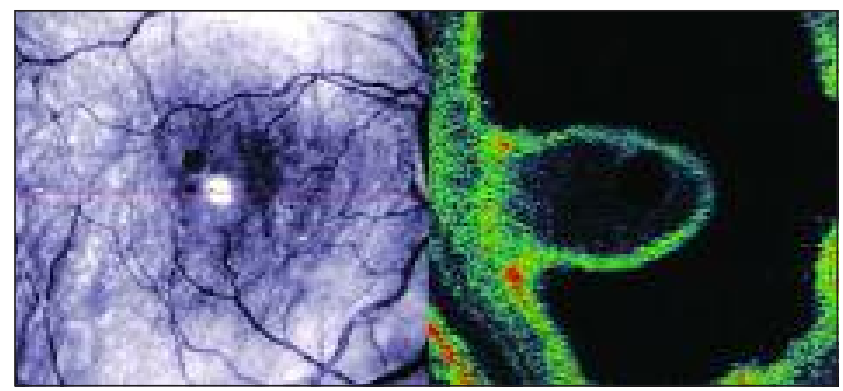

Figura 3: OCT/SLO do OD demonstrand o DVP

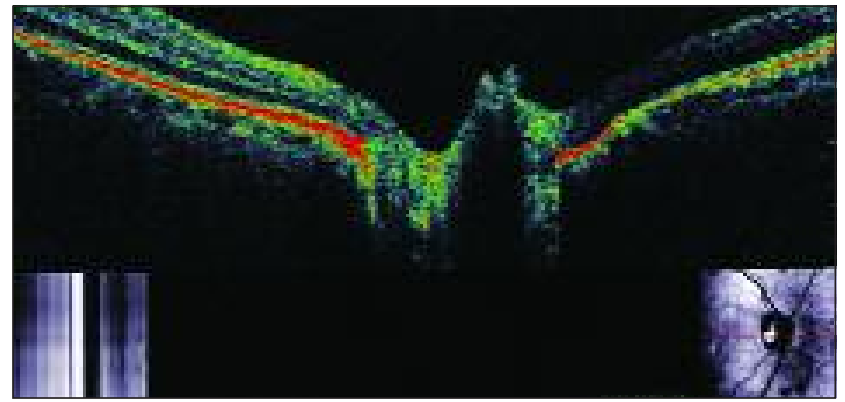

Figuras 5 e 6: OCT de papila e de mácula do OD dentro da normalidade

além da hemorragia do disco óptico no OD. Neste sentido, foram realizados exames de perimetria computadorizada e OCT para investigação de possível dano glaucomatoso associado, o que não foi observado durante o seguimento.

Neste contexto, optou-se pela observação clínica do caso que revelou regressão da hemorragia de disco óptico sem recidiva e a permanência dos floaters que são compatíveis com a condensação vítrea sobre o eixo visual.

O diagnóstico das alterações na interface vítreoretiniana está entre as mais importantes contribuições

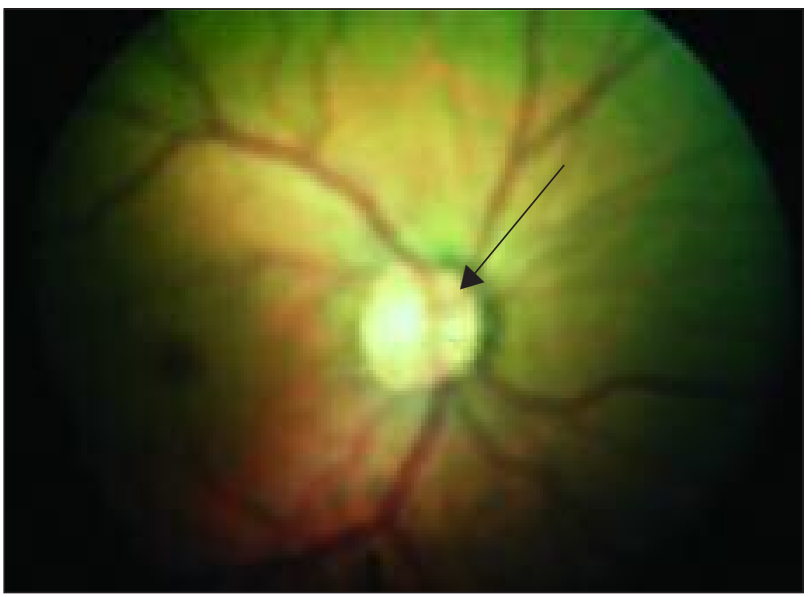

Figura 2: Anel de Weiss

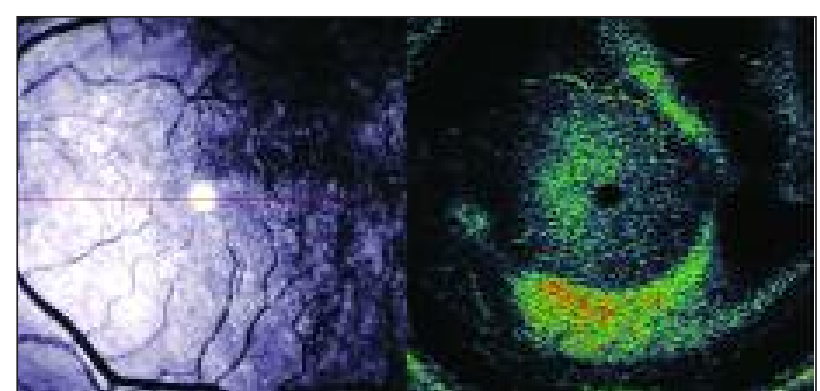

Figura 4: OCT/SLO do OE demonstrando vítreo posterior aderido

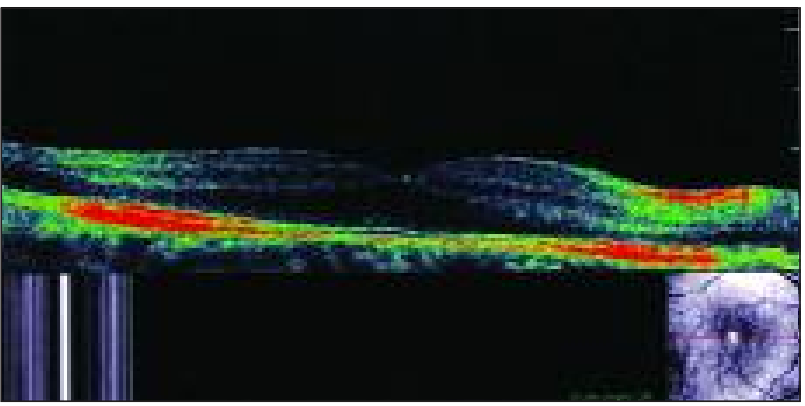

disponibilizadas à oftalmologia clínica pelo OCT e o avanço da integração de um sistema de imagem multiplanar oferecido pelo SLO/OCT parece ser bastante promissor, pois possibilita a reconstrução da imagem retiniana tridimensionalmente, contribuindo como um avançado método alternativo e eficaz no diagnóstico e seguimento do descolamento vítreo.

Desta forma, o Oftalmologista deve estar atento aos pacientes com sintomatologia de floaters e fotopsias, acompanhando-os com freqüência e os orientando quanto aos riscos das possíveis complicações. 


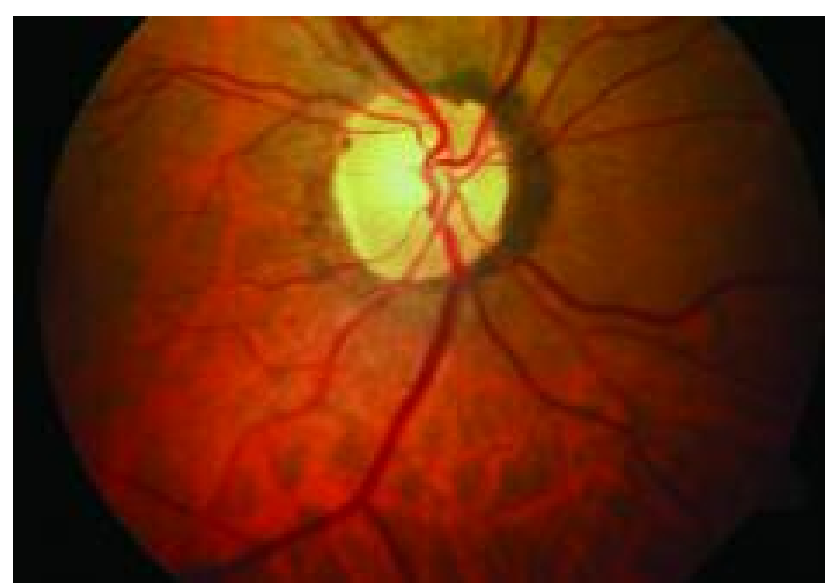

Figura 7: Disco óptico após 6 horas de evolução

\section{Abstract}

To describe the diagnosis and management of a optic disc haemorrhage probably caused by an acute and spontaneous posterior vitreous detachment. A single casereport of an optic disc haemorrhage probably caused by an spontaneous posterior vitreous detachment, in order to discuss the diagnosis, management and prognosis. Optic disc haemorrhage regression was observed after 6 hours of evolution without relapsing signals during the subsequent registers. The Scanning Laser Ophthalmoscopy revealed as an advanced alternative and efficient method in the vitreous detachment diagnosis and follow up.
Keywords: Vitreous detachment; Retinal hemorrhage; Optic disk/blood supply; Ophthalmoscopy / methods; Case reports [Publication type]

\section{RefERÊNCIAS}

1. Suzuki H. Maculopatias por alterações da interface vitreorretiniana. In: Bonomo PP, Cunha SL, editores. Doenças da mácula. São Paulo: Roca; 1993. p. 215-9.

2. Foos RY. Posterior vitreous detachment. Trans Am Acad Ophthalmol Otolaryngol. 1972; 76(2):480-97.

3. Ko TH, Fujimoto JG, Duker JS, Paunescu LA, Drexler W, Baumal $\mathrm{CR}$, et al. Comparison of ultrahigh- and standard-resolution optical coherence tomography for imaging macular hole pathology and repair. Ophthalmology. 2004; 111(11): 2033-43.

4. Rosen RB, Podoleano AG, Dunne S, Garcia PM. Optical coherence tomography ophthalmoscopy. In: Ciulla T, Regillo C, Harris A, editors. Retina and optic nerve imaging. Philadelphia: Lippincott Williams \& Wilkins; 2003. p. 119-36.

5. Yannuzzi LA, Ober MD, Slakter JS, Spaide RF, Fisher YL, Flower RW, Rosen RB. Ophthalmic fundus imaging: today and beyond. Am J Ophthalmol. 2004; 137(3): 511-24.

6. Sugiyama K, Ishida K, Uchida H, Yamamoto T, Kitazawa Y. Disc haemorrhages in normal tension glaucoma. Asian J Ophthalmol. 2000; 2(3):3-8.

7. McDonald HR, Johnson RN, Schatz H. Surgical results in the vitreomacular traction syndrome. Ophthalmology. 1994; 101(8):1397-402; discussion 1403.

ENDEREÇO PARA CORRESPONDÊNCIA:

Rodrigo Interlandi Angelucci

Av. Bosque da Saúde 546, apto. 151

CEP 04142-081 - São Paulo - SP

E-mail: rodrigoangelucci@yahoo.com.br 